Cahiers d'études italiennes

$7 \mid 2008$

NOVECENTO... E DINTORNI

Images littéraires de la société contemporaine (3)

\title{
Images et formes de la différence identitaire et insulaire chez Marcello Fois
}

\section{Margherita Marras}

\section{OpenEdition}

Journals

Édition électronique

URL : http://journals.openedition.org/cei/915

DOI : $10.4000 /$ cei.915

ISSN : 2260-779X

\section{Éditeur}

UGA Éditions/Université Grenoble Alpes

\section{Édition imprimée}

Date de publication : 15 mai 2008

Pagination : 113-124

ISBN : 978-2-84310-121-2

ISSN : 1770-9571

Référence électronique

Margherita Marras, «Images et formes de la différence identitaire et insulaire chez Marcello Fois », Cahiers d'études italiennes [En ligne], 7 | 2008, mis en ligne le 15 novembre 2009, consulté le 19 avril 2019. URL : http://journals.openedition.org/cei/915 ; DOI : 10.4000/cei.915 


\title{
IMAGES ET FORMES DE LA DIFFÉRENCE IDENTITAIRE ET INSULAIRE CHEZ MARCELLO FOIS
}

\author{
Margherita Marras \\ Université d'Avignon
}

Parler des images et formes de la différence dans les ouvrages sardes de Marcello Fois conduit inévitablement à s'arrêter sur les concepts de sardité et d'insularité. Ainsi en va-t-il dans les deux trilogies (bientôt tétralogies selon les projets de l'auteur), l'une de genre historique (fin XIX) avec Sempre caro $^{1^{*}}$, Sangue dal cielo ${ }^{2}$, L'altro mondo ${ }^{3}$, l'autre située à l'époque contemporaine (dernières années du XXe), composée de Ferro recente ${ }^{4}$, Meglio morti ${ }^{5}$ et Dura madre ${ }^{6}$. Si les temps de la représentation sont différents, on ne peut en dire autant pour l'espace, la Sardaigne, qui reste le point constant de référence. Dans les deux cas est évidente la préférence du romancier de Nuoro pour les époques de transition, de passage, qui correspondent à des moments cruciaux du passé et du présent de l'île. Indéniablement les deux trilogies présentent des éléments de continuité laissant à penser qu'elles peuvent être le fruit d'un même projet: leur complémentarité se lit au travers des éléments constitutifs de l'univers insulaire des romanciers contemporains reliés aux dérives politiques et socio-culturelles rapportées dans les œuvres historiques en une relation cause-conséquence indélébile.

Un autre facteur commun aux romans des deux trilogies est le genre policier dont la particularité exprime l'éclectisme de Marcello Fois et sa fructueuse relation avec les centres les plus importants du roman policier italien (il est l'un des principaux représentants du Gruppo 13 de Bologne), comme avec la culture littéraire sarde caractérisée, à partir des années 80 , par l'apparition et l'affirmation de nouvelles formes du récit qui ont attiré, au cours de la décennie suivante, l'attention d'un public national et international. Marcello Fois a sans aucun doute été l'un des principaux artisans de cette "nouvelle vague ", surtout en sa qualité de chef de file du policier sarde. Si le contexte insulaire n'est pas une constante dans la production

* Notes p. 123.

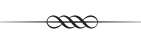

Cahiers d'études italiennes, $\mathrm{n}^{\circ}$ 7, 2008, p-113-124. 113 
de l'auteur, les formes et les nombreuses images de la différence présentent, dans les deux trilogies, un rapport direct avec la condition historiquement, socialement et culturellement périphérique de la Sardaigne. Cette constance dans la méthode permet d'établir des similitudes parlantes avec des littératures et des cultures enracinées en d'autres lieux et en d'autres contextes à forte spécificité et qui illustrent une vérité fondamentale: la diversité ne se donne pas à lire seulement selon l'optique de la singularité absolue et de la distanciation, mais aussi selon celle du partage et du rapprochement.

On a là l'une des raisons justifiant une lecture croisée entre les œuvres de Marcello Fois et certaines théories littéraires, récemment dites postcoloniales. Cette juxtaposition pourrait sembler étrange, surtout en raison de la signification historique couramment attribuée à l'expression postcolonial, souvent utilisée " de manières différentes et pas toujours cohérentes $^{7}$ ». Naturellement elle est prise ici dans une acception limitée aux œuvres narratives et aux théories exprimées par les écrivains qui se font voix d'une minorité, qui se distinguent par un discours critique exposant la conflictualité des relations entre centres du pouvoir et réalités périphériques, par l'importance de leur réflexion sur les dynamiques profondes de la diversité (qu'elles soient de nature historique, économique, socio-culturelle, psychologique ou linguistique) et, plus généralement, qui se réferent à " une idée globale de l'Occident ${ }^{8}$ ».

Qui connaît l'histoire et la littérature sardes reconnaîtra sans difficulté dans ces références les traits distinctifs de nombre de thématiques identitaires fréquentes dans la littérature narrative de l'île et dont témoignent aussi les romans de Marcello Fois.

Tenant compte de l'ampleur du sujet, nous limiterons la comparaison au phénomène de l'antillanité telle que la repropose et la discute Édouard Glissant et à la relation entre créolité et écriture élaborée par Jean Bernabé, Raphaël Confiant er Patrick Chamoiseau, dans ce que l'on peut considérer comme un manifeste: l'Éloge de la créolité'.

\section{Images de la différence insulaire et identitaire}

L'une des bases et des constantes pour la conceptualisation et la formalisation littéraires, aussi bien dans les ouvrages historiques de Fois que dans les théories des écrivains créoles, est le paradigme centre-périphérie dont 
naît une série d'images que l'on peut ranger selon deux typologies principales: les images discursives et les images poétiques (ou construites sur des bases analogiques et métaphoriques). Il ne s'agit pas en réalité d'une séparation draconienne mais plutôt d'une répartition aux limites floues: ces deux typologies apparaissent, en effet, fonctionnelles l'une à l'autre et c'est dans leur continuelle intersection que se manifeste le rapport colonial qui lie la Sardaigne au pouvoir central, noyau fondateur et fil conducteur du récit historique sarde chez l'écrivain de Nuoro.

Très nombreux sont les rappels de cette subalternité: l'île est présentée comme " canile di Roma ${ }^{10}$ ", elle est définie terre d'exil et de "selvaggi ${ }^{11}$ " et l'altérité insulaire est perçue par l'état italien comme sous-culture:

Non siamo cittadini qualunque, non italiani come gli altri. Noi siamo carne da lavoro e cani da guerra. Una cosa l'abbiamo capita subito [...] di quello che siamo, di quello che siamo stati, di quello che saremo, non importa niente a nessuno ${ }^{12}$.

Fort éloquente est à ce propos la représentation inscrite dans les lois spéciales, promulguées par le gouvernement Pelloux (1898-1899) pour garantir la sécurité publique en Sardaigne et combattre le banditisme, auxquelles Marcello Fois se réfere pour ce qui concerne leur application. Des lois que l'avocat Bustianu ${ }^{13}$, serial detective de la trilogie historique, critique en de véritables réquisitoires pour leur caractère punitif ${ }^{14}$, pour leur inefficacité et, surtout, pour la nocivité de leurs conséquences: l'aggravation de la fracture entre l'île et le continent et de la défiance des Sardes face à l'État italien et à la Justice ${ }^{15}$. Si elles sont évoquées dans les trois romans historiques, c'est dans L'altro mondo que les lois spéciales acquièrent une place centrale: images, dialogues et considérations diverses deviennent illustration d'une véritable " guerre ", conduite par les forces de l'ordre et d'importants détachements militaires expédiés sur la terre sarde pour opérer des perquisitions et des arrestations en masse des parents des fugitifs (y compris les femmes et les enfants), dans le but de contraindre ces derniers à se rendre.

En fait, chez de Fois, ces lois sont les instruments de complots et de chantages, tant privés que publics, aux dépens de sujets faibles et marginaux (les Sardes socialement et économiquement les plus fragiles ${ }^{16}$ ). Loin d'être le moyen de combattre le crime, elles sont bien plutôt celui par lequel il se perpétue en raison de leur évidente utilisation comme très efficace instrument de l'affirmation de logiques d'état prévaricatrices et d'exécution des ignobles actions prévues dans le protocole "Altro mondo ", comme on le découvre dans les pages du roman homonyme. Ce protocole, charpente et coeur du texte, consiste en une préparation expérimen- 
tale à une " guerre totale» (p. 172) que les dirigeants italiens considéraient stratégiquement nécessaire pour prévenir de nouvelles défaites après le désastre de Adua (1896). Bien que sa réalisation ait été officiellement abandonnée en raison de "l'impossibilità di trovare uno spazio adatto " (p. 172) sur le territoire national et du refus de quelques députés, le projet est secrètement mis en œuvre en Sardaigne. L'intrigue, les crimes et l'enquête du roman sont entièrement liés à la mise en œuvre du protocole, source d'images discursives et figuratives tragiquement poétiques où l'île, éventrée et anéantie, devient le décor de la transposition métaphorique d'une histoire d'abus et d'injustices. On le voit dans le climat de chaos, le bouleversement et la transformation de la "zona franca " (p. 81), zone d'expérimentation de l'" altro mondo » (p. 81), en " un paradiso che sa d'inferno » (p. 81) et de mort:

Quello che vedono ${ }^{18}$ sono scheletri immersi nella poltiglia delle loro carcasse. I cinghiali sono morti e sono morti i corvi che di loro si sono pasciuti. Ma nel marasma fetido sono visibili piume di tortora e pelli di donnola. Ghigni di lepre emergono dalla mucillagine sanguinolenta. (p. 84)

Autant d'images qui apportent une nouvelle signification au caractère plurisémantique et à la valeur dichotomique du titre du roman :

Altro mondo evoca l'opposizione dell'isola [...] ad un Altrove. [...] Altro mondo implica una relazione con l'alterità [...]. Altro mondo sottintende l'immagine di un'isola primitiva caratterizzata dall' 'isolazionismo e dall' 'isolamento, risultante della sua separatezza geografica e delle sue differenze socioculturali ${ }^{19}$.

La matière historique est pour Marcello Fois une source inépuisable d'inspiration. Bien qu'il ne s'agisse pas d'un élément fondamental des intrigues, il est utile de rappeler l'évocation, dans chacun des trois romans, de l'Édit des enclosures, l'un des événements qui a le plus fortement marqué le passé de la Sardaigne et que les narrateurs insulaires ne cessent de reprendre dans leurs œuvres de fiction. Promulgué en 1820 par le gouvernement piémontais, l'Édit accordait aux municipalités et aux particuliers le droit d'enclore les terres incultes, jusqu'à cette date librement utilisées par les paysans et les bergers. Fois s'y réfere au travers d'une vigoureuse polémique en défense de l'île et en dénonciation d'une histoire de l'absence ${ }^{19}$ tout en opérant, au niveau structurel, un mouvement à rebours qui interrompt le présent de la narration. Cette technique a pour conséquence de créer un effet de redondance: dans les récits du romancier de Nuoro, la référence à l'Édit, présenté comme un droit "imposé ", établit un lien de continuité entre les politiques d'oppression précédentes et les nombreuses actions coercitives menées par le pouvoir central dans la 
Sardaigne de la fin du XIX ${ }^{e}$ siècle. Dans Sangue dal cielo ${ }^{20}$ elle est le point de départ d'une impressionnante représentation apocalyptique construite sur une succession tragique d'événements impliquant le site insulaire dans son intégralité:

Alla fine di ottobre del 1820, dopo nove giorni e nove notti di pioggia da annegare il cristiano e le talpe sotto terra, dal sud si era fatto largo a furia di spinte un alito unticcio e caldissimo, si era aperto un corridoio rotolando verso le Barbagie. E le montagne cominciarono a sudare sangue. [...] Proprio nell'ottobre del'20. L'anno dell'Editto sulla chiusura delle terre comuni. Che allora pareva un segnale chiaro dell'ira della povera gente. La zaffata micidiale arrivò con i banditori del re che annunciavano i muri a secco. L'editto arrivò accompagnato da un fetore ferruginoso di acqua stagnate. E intrise valli e montagne di sangue. La ricchezza non era l'oro, e nemmanco il pane, ma pietre su pietre per cingere i terreni e braccia per costruirli: questo diceva l'editto. E al sangue dal cielo si unì il sangue degli uomini, il massacro delle tanche. Che se giravi il cielo con la terra era lo stesso : gli uomini e le nuvole lottavano allo stesso modo. E sarebbe stato uguale persino non essere nati mai in quella terra dove l'alto e il basso di confondevano. (p. 72-73)

Les lois spéciales, le protocole «Altro mondo » et l'Édit des enclosures ne forment qu'une partie des nombreuses références historiques qui, traduites en récit par Marcello Fois, conduisent à une représentation de l'histoire qui ne peut être perçue comme développement diachronique d'événements, mais bien plutôt comme circularité et répétition éternelle d'actes menant à l'anéantissement de l'île. Un concept d'une histoire nonhistoire récurrent dans le roman sarde (qu'on retrouve aussi chez les auteurs italiens du Mezzogiorno) et présente dans les œuvres et les théories des auteurs antillais comme Édouard Glissant qui, dans Le discours antillais, parle, en référence à la Martinique, d'une " "non-histoire" syncopée $^{21}$ ». Cependant, si la similitude entre ces deux auteurs insulaires est évidente dans le regard porté sur leur passé, il n'en faut pas moins préciser que le parcours indiqué par Glissant pour l'explication du phénomène n'est pas, comme c'est le cas pour la Sardaigne de Fois, la déstructuration de codes autochtones et identitaires mais plutôt l'absence et l'impossibilité de leur construction.

Un autre point évident de contact entre les écrivains antillais et le Sarde Marcello Fois se manifeste dans l'importance attribuée au paysage. Dans les romans de la trilogie historique les éléments naturels, les espaces et les lieux les plus reculés sont soumis à un processus d'anthropomorphisation et à l'insertion de détails liés aussi bien à la culture qu'à l'histoire sardes. L'espace devient ainsi un personnage en soi qui subit, comme les 
hommes, le poids des contradictions du passé et du présent se transformant, comme l'écrivent aussi les théoriciens de la créolité 22 , en un témoignage tangible de la mémoire collective et du bagage d'émotions et de sensations de ses habitants. Il en va de même dans les romans situés à l'époque contemporaine où les blessures, la dénaturation et les transformations du paysage et de l'espace insulaires deviennent le miroir fidèle des bouleversements advenus dans la société de Nuoro et confirment son incapacité à soutenir la confrontation avec la modernité. Dans Ferro recente $^{23}$, les gens de Nuoro, qui n'arrivent pas à proposer au « futuro un compromesso dignitoso " (p. 50) et à préserver ce qu'il y avait de bon dans leurs traditions: du passé, ils ont conservé le pire. C'est ce que démontre la représentation constante, réalisée par la triade thématique (soumission, fatalisme et servilisme acritique) souvent utilisée par l'auteur, des tares et de maux endémiques. Coupables d'avoir laissé le temps "scorrere addosso " (p. 51), ils sont responsables du " livellamento feroce, vorace " (p. 51) qui a frappé toute chose, transformant la Sardaigne en

un organismo privo di anticorpi : restava il folclore, l'esibizione di riti non più rituali, senza significato, restava quel collegamento inconscio con valori estranei e fatali. Restava la violenza. (p. 51)

Et c'est ainsi que les personnages insulaires de Fois, dans la trilogie contemporaine, n'évaluent plus leur propre altérité en fonction de l'Autre, du non-insulaire - comme c'est en revanche le cas dans la trilogie historique - mais par rapport au sentiment d'être étrangers à leur propre vie, par rapport à un dépaysement qui leur interdit de se repérer dans leur propre espace-temps. Et la dégradation du paysage devient le révélateur d'une géographie complexe de la négation qui remodèle et répète toutes les dérives et les corruptions morales, politiques et matérielles qui ont frappé l'île: Nuoro est une ville explosée ${ }^{24}$, le royaume de la spéculation immobiliaire, des constructions abusives suivies de régularisation ${ }^{25}$, c'est le lieu de l'inachevé et du non sens, à l'image des chantiers jamais achevés qui la caractérisent.

\section{Pratiques d'écriture: oralité et oraliture}

C'est dans les pratiques d'écritures liées, dans leur forme, à l'oralité que la ressemblance entre Marcello Fois et les théoriciens antillais se montre la plus forte. L'oralité est sans aucun doute l'une des bases de la construction et de la représentation du site insulaire dans la trilogie historique et, 
quoique de manière moins invasive, dans Dura madre ${ }^{26}$. Elle se manifeste dans le sens le plus traditionnel, à savoir dans la transposition à l'écrit de formules linguistiques d'expression verbale et de registres oraux, reconductibles d'un côté à l'usage de formes orales générales (non circonscrites au monde sarde) et, de l'autre, à l'intégration d'expressions relevant d'une norme de récit parlé de matrice sarde: on note en effet d'évidentes interférences du sarde aux niveaux structurel, morphosyntaxique et lexical. Cette procédure, visant à la recherche d'une "fidélité " expressive, se retrouve d'ailleurs aussi bien dans le discours indirect que dans le discours direct. De fait, la mixité linguistique est aussi une prérogative du narrateur, particulièrement de celui qui, dans la pluralité des voix narrantes qui peuplent les romans de Fois, assume le rôle de conteur de la communauté nuoraise, avec pour fonction le témoignage et la transmission. À l'instar des personnages d'extraction populaire, son langage est imprégné de régionalismes syntaxiques ${ }^{27}$, pléonasmes ${ }^{28}$, expressions et greffons sardes ${ }^{29}$ qui s'intègrent au texte italien sans que l'auteur formalise, par la ponctuation ou l'emploi de caractères différents, le changement de registre. Ainsi se définit naturellement une évolution de la phrase qui, outre qu'elle exprime une incontestable cohérence avec le monde raconté, tend à focaliser le paradoxe existant entre les représentations de la diversité culturelle sarde et les prétentions du pouvoir central qui met en ouvre une expéditive et violente assimilation politique et culturelle de la Sardaigne. Dans cette opposition apparaissent toutes les contradictions du processus $\mathrm{d}^{\prime}$ " italianisation » forcée ${ }^{30}$. Mais la signification inhérente aux éléments linguistiques caractérisant nombre de personnages s'applique aussi à l'importance de l'oralité dans la culture sarde, objet, dans Sangue dal cielo, d'une véritable défense théorique. En effet, un code expressif argumentatif, propre à la tradition narrative de l'île, sert de base à la réflexion polémique, conduite par Bustianu, contre les cultures de conquête et le concept d'unicité et exclusivité culturelles. C'est une mise en accusation des pratiques coloniales appliquées en "India o America che fosse " (p. 98), avec une référence explicite à Christophe Colomb et à son attitude envers les indigènes qu'il décide de transporter sur l'une de ses caravelles " per farli vedere al re » (p. 98). Son récit met en relief la préoccupation de Colomb qui craint qu'au souverain, comme à " tutta la cultura, l'unica cultura che esso incarnava " (p. 98), puisse déplaire que ces indigènes n'aient pas la moindre connaissance de l'espagnol. Il se fait donc précéder par une missive dans laquelle il précise "porterò sei di questi uomini alle Vostre Altezze, così che possano imparare a parlare" (p. 98). La citation de Bustianu est suivie d'un commentaire au contenu révélateur: 
"Capisce? Incalzai. "Non ha detto: così che possano imparare a parlare lo spagnolo; ha detto così : che possano imparare a parlare" "(p. 98-99).

Cette explication de Bustianu entraîne implicitement un déplacement vers la situation et l'histoire de l'île, en cela qu'elle se présente comme un élément de transition vers une réflexion successive qui souligne la priorité absolue de la parole dans la société sarde, laquelle a construit sur le récit oral sa mémoire propre:

Le parole hanno costruito ogni singolo meccanismo della nostra società : noi con le nostre parole abbiamo costruito civiltà di pietra levigata e codici universali. Non $a$ parole. La scrittura è arrivata dopo dalle navi fenicie e romane. Ed era scandalosa. (p. 99)

À partir de ces références spatio-temporelles croisées, du transfert dans le présent des situations passées, il est clair que la signification de cette défense théorique de l'oralité s'étend à toutes les réalités marginales ayant subi, ou subissant, abus et violations de leurs particularismes culturels. Ainsi apparaît clairement la nécessité de l'identité relation, concept par lequel Édouard Glissant, dans Le discours antillais, affirme qu'il ne peut exister d'intégration sans le respect des diversités ethniques et culturelles.

De la même façon, dans Dura madre, l'auteur joue à la fois sur les deux tableaux du style et de la réalité, avec des conséquences évidentes sur la structure narrative. Dans la société nuoraise de Marcello Fois, aliénée et déstructurée, se côtoient une multitude de codes expressifs (qui vont des codes moyens - sobres et linéaires - à la violence langagière des jeunes, tel celui de la Cosmo good) sans oublier une série de formules et de matériels linguistiques (actes officiels, administratifs, etc.) dont l'utilisation semble viser la mise en évidence des aspects nuisibles de la neutralisation dont a été victime la Sardaigne. Il faut sans doute lire en ce sens la présence plus rare dans Dura Madre, par rapport aux trois romans historiques, de régionalismes syntaxiques, de greffons, de termes sardes italianisés, qui de plus sont signalés par l'italique, souvent suivi par une traduction ${ }^{31}$ ou une explication $^{32}$ en langue italienne. Le naturel expressif, caractéristique de la trilogie historique, est donc remplacé par des formalisations et formulations précises qui transforment les références linguistiques au sarde en bribes de souvenirs opérant sur le plan de la composition pour faire apparaître les résultats de la brutale superposition aux codes culturels de l'île d'autres codes, qui lui sont " estranei e fatali ${ }^{33}$ ".

Les choix plurilinguistiques que propose Marcello Fois ne représentent, cependant, que l'un des nombreux processus de la bien plus com- 
plexe " oralisation de l'écrit ${ }^{34}$ " (confrontation entre la pratique d'écriture et l'oralité) dont parle Glissant dans son Discours antillais. Dans Dura Madre, par exemple, on retrouve une série de mécanismes typiques de l'oralité au sein même de la structure du récit. Ainsi en va-t-il explicitement de ce que le roman définit comme des métaphores, qui renvoient à des termes de l'ancienne topographie de Nuoro (entre autres, un terrain dit "Mortu s'omine ») ou à des expressions en usage dans la quotidienneté linguistique des personnages ("Il cane di Fronteddu»). Ces métaphores sont à l'origine d'une véritable transcription et/ou recréation de paraboles, autrement dit d'histoires, intégrées au récit, dont la fonction de comparaison illustre un enseignement par analogie. C'est ce que le juge sarde Salvatore Corona explique au commissaire Sanuti, romagnol fraîchement arrivé en Sardaigne

Ma come, non ha capito? La metafora, intendo, o se preferisce la parabola. Qui le parabole si mangiano a colazione: lei dica una parola, noi abbiamo una parabola o, più semplicemente, una rima corrispondente. (p. 41)

Leur emploi renvoie à la double particularité de ce code distinctif: si en effet, d'un côté, paraboles et métaphores ont une fonction explicative, de l'autre elles présentent une valeur évocative qui s'étend à un système de relations spécifique de la communauté nuoraise. Techniquement cette pratique de transposition et transcription de paraboles et de métaphores est reconnaissable et identifiable dans l'oraliture, terme créé dans les années 70 par l'écrivain haïtien Ernst Mirville, pour désigner la reproduction dans l'écriture de matériaux préexistant dans la culture et la tradition orale: légendes, récits populaires, proverbes, etc. Bien évidemment, ces procédés d'oraliture, chez Marcello Fois comme chez les Antillais, vont bien au-delà de la simple technicité. Les auteurs de l'Éloge de la créolité35 ont théorisé à diverses reprises l'utilisation de l'oralité en littérature, qu'ils définissent aussi comme " enracinement dans l'oral (p. 33), comme élément lié à leur survie culturelle et identitaire:

l'oralité est notre intelligence, elle est notre lecture de ce monde, le tâtonnement, aveugle encore, de notre complexité. L'oralité créole, même contrariée dans son expression esthétique, recèle un système de contre-valeurs, une contre-culture; elle porte témoignage du génie ordinaire appliqué à la résistance, dévoué à la survie. (p. 33-34)

La valeur symbolique et démonstrative attribuée à l'oraliture par le romancier sarde semble aller en ce sens. L'interpréter ainsi permettrait par ailleurs de résoudre ce qui pourrait apparaître, dans Dura madre, comme une contradiction de fond. On a dit que l'une des caractéristiques stylis- 
tiques du roman repose sur la pluralité des registres linguistiques et la faible présence du sarde, toutes deux emblématiques de la perte des valeurs culturelles liées à la tradition insulaire. Avec les techniques d'écriture liées à l'oraliture, l'auteur semble en revanche revitaliser la tradition orale sarde précisément par l'emploi de particularismes culturels et sociolinguistiques liés au monde même dont il raconte la fin. Il est délicat d'expliquer la cause et les raisons de ces choix d'auteur, mais si l'on s'en tient au texte, l'effet produit est tel que les procédés inhérents à l'oraliture fonctionnent comme une sorte d'antidote littéraire à la représentation d'une tradition sarde désormais domptée et transformée en folklore, une démonstration pratique de résistance aux dangers de l'assimilation et de l'aliénation culturelle. Il en résulte une reconstruction convaincante, quoique romancée, de la mémoire historique d'une île à la fois particulière et universelle. On assiste en effet dans les romans insulaires de Marcello Fois à un véritable voyage dans la particularité sarde qui ne se traduit jamais en une exaltation de principes liés au localisme ou à des célébrations " nombrilistes ». La réutilisation des matériaux de la tradition orale, sève nourricière de ses œuvres de fiction, devient le lumineux témoignage de la capacité des petites réalités, égales en cela aux grandes, à enrichir un projet littéraire, en faisant " corps avec le monde ${ }^{36}$ ", ainsi que le soulignent les théoriciens antillais dans l'Éloge de la créolité. Là est le sens (lié à une dynamique littéraire et identitaire) du savoir " subsister dans la diversité » (p. 53) qui implique que l'écrivain se reconnaisse, se structure et se préserve mais sache aussi se placer « en situation d'irrruption ${ }^{37}$ » (p. 42) en renouvelant les modèles littéraires de la tradition pour s'inscrire dans la modernité (p. 36). Marcello Fois est certainement un écrivain de son temps: il réutilise et dépasse la tradition en imprégnant son écriture de techniques et de styles d'expression d'orientation progressiste et en recherchant constamment de nouvelles esthétiques.

L'auteur nuorais et les écrivains post-coloniaux sont les chantres d'un art capable de métaboliser le passé pour une nécessaire projection dans l'avenir et, surtout, ils démontrent combien la diversité culturelle dans l'œuvre d'un narrateur est et doit être une valeur poétique ajoutée. Ce qui a aujourd'hui plus de sens encore qu'hier, en cela que les œuvres d'écrivains tels que Marcello Fois nous portent à une réflexion sur l'adaptabilité du roman contemporain, et à reconsidérer ses nouvelles potentialités non plus, ou non seulement, dans une dimension nationale mais à travers une nécessaire confrontation avec l'actuel contexte multiculturel. 


\section{Notes}

1. M. Fois, Sempre caro, Nuoro, Il Maestrale, 1998.

2. M. Fois, Sangue dal cielo, Milano, Il Maestrale-Frassinelli, 1999.

3. M. Fois, L'altro mondo, Milano, Il Maestrale-Frassinelli, 2002.

4. M. Fois, Ferro recente, Bologna, Granata Press, 1992.

5. M. Fois, Meglio morti, Torino, Einaudi, 2000.

6. M. Fois, Dura madre, Torino, Einaudi, 2001.

7. F. Neri, "Multiculturalismo, studi postcoloniali e decolonizzazione", Introduzione alla letteratura comparata, A. Gnisci, Milan, Mondadori, 1999, p. 267.

8. Voir M. Pala, «The return of the native. L'idea di minoranza in alcune letterature del commonwealth secondo la critica post-coloniale», Letterature marginali/emarginnate, Eudossia I, 2002, p. 49.

9. Conférence prononcée le dimanche 22 mai 1988 au Festival caraïbe de la Seine-Saint-Denis. Maintenant in J. Bernabé, P. Chamoiseau, R. Confiant, Éloge de la créolité [1989], Paris, Gallimard, 1993.

10. M. Fois, Altro mondo, op. cit., p. 161.

11. M. Fois, Sempre caro, op. cit., p. 63.

12. Ibid., p. 62.

13. Pour sa construction romanesque du personnage de Bustianu, Fois s'est inspiré de l'avocat et poète nuorais Sebastiano Satta (1867-1914).

14. «Non ne hanno fatte abbastanza di leggi speciali ? Altroché se ne hanno fatte! Tutte per noi le hanno fatte ! E tutte per punire ! Le abbiamo viste lo scorso maggio in azione le leggi speciali ! Arresti in massa. Quanti erano gli arrestati ? Quattrocento ? Perquisizioni arbitrarie ; bambini e persino donne incinte tirati giù dal letto, ammassati sul sagrato! Tutti delinquenti, tutti colpevoli !», M. Fois, Sangue dal cielo, op. cit., p. 32.

15. Avec ces lois, comme le fait remarquer Bustianu, l'État et la justice se placent « sullo stesso piano, ma io dico peggiore, di colui che l'offende [il bandito] ", M. Fois, Altro mondo, op. cit., p. 67.

16. En exploitant leurs faiblesses. La complicité de ceux des Sardes qui se sont prêtés à la politique de l'"Altro mondo " est âprement critiquée par Bustianu qui compare l'action de Seddone à une guerre entre gueux : "Come tutti i servi sciocchi che in nome di un progetto che sempre li sovrasta, e mai li riguarda, si prestano alla lotta tra pezzenti. Perché sia chiaro che hanno perso due volte : hanno perso la vita e hanno perso la dignità », M. Fois, Ibid., p. 196.

17. Il s'agit de Bustianu, Zenobi et du maréchal des logis Poli : venus dans la zone franche à la recherche d'indices pouvant éclairer les causes et les mobiles de l'exécution de Elena Seddone, ils découvrent à l'entrée de la caverne ce macabre spectacle.

18. M. Marras, "Paradossi e particolarità dello spazio insulare in Marcello Fois ", Mélanges offerts à Marie-Hélène Caspar. Littérature italienne contemporaine, CRIX, Novembre 2005, p. 556.

19. Voir M. Fois, Sempre caro, op. cit., p. 108.

20. M. Fois, Sangue dal cielo, op. cit.

21. É. Glissant, Le discours antillais [1981], Paris, Gallimard, 1997, p. 325.

22. Voir J. Bernabé, P. Chamoiseau, R. Confiant, Eloge de la créolité, op. cit., p. 38

23. M. Fois, Ferro recente, op. cit.

24. " città che scappava da tutti i lati colando dall'altipiano come una zuppa che, per troppo bollore, fosse debordata dal recipiente ", M. Fois, Meglio morti, op. cit., p. 120.

25. M. Fois, Meglio morti, op. cit., p. 67, p. 178 ; M. Fois, Ferro recente, op. cit., p. 101 ; M. Fois, Dura madre, op. cit., p. 14, p. 44.

26. Comme dans la trilogie historique, des formes grammaticales inspirées du sarde et des mots ou des constructions sardes sont utilisés, dans Dura madre, par les nombreux narrateurs communautaires et par les personnages les plus âgés, ceux qui représentent le mieux l'ancien monde : la mère et le père Marongiu, Antonio Lilliu, le gardien du cimetière.

27. Récurrence du « che » dont l'emploi reproduit souvent l'usage adverbial du « Ca » (parce 


\section{MARGHERITA MARRAS}

que) sarde de dérivation latine (quia). Voir, entre autres, M. Fois, Sempre caro, op. cit., p. 19 ; M. Fois, Sangue dal cielo, op. cit., p. 45.

28. Voir, entre autres, M. Fois, Sempre caro, op. cit., p. 15, p. 60.

29. Voir ibid., p. 26-27, p. 36, p. 45, p. 46, p. 112-113, p. 132.

30. Comme on peut le déduire de cette réflexion de Bustianu dans Sangue dal cielo (op. cit.) : "L'Italia è una nazione ancora troppo giovane : deve passare il suo tempo prima che riusciamo a parlare la stessa lingua. E non mi riferisco al linguaggio in se stesso, mi riferisco a quella cultura che, nel bene o nel male, è un nostro patrimonio comune. Non avevamo mica l'anello al naso quando l'hanno fatta quest'Italia. Questo dico : ci lascino il tempo di stabilire come vogliamo starci in questa nazione. Penso che saremmo italiani migliori se ci fosse permesso di entrarci da Sardi in questa nazione ", p. 32.

31. Voir M. Fois, Dura madre, op. cit., p. 31, p. 32, p. 120, p. 126, p. 127, p. 137.

32. Voir, ibid., p. 45.

33. M. Fois, Ferro recente, p. 51.

34. É. Glissant, Le discours antillais, op. cit., p. 451.

35. J. Bernabé, P. Chamoiseau, R. Confiant, Éloge de la créolité, op. cit.

36. Ibid., p. 39.

37. Concept utilisé par É. Glissant, puis repris par J. Bernabé, P. Chamoiseau, R. Confiant, dans Éloge de la créolité. 